\title{
Perfil leucocitário na faixa etária \\ de 15 a 22 anos em função do nível de condicionamento aeróbico
}

\author{
Artigo Original
}

\section{Marco Aurélio Dalfior}

Universidade Castelo Branco - UCB - Brasil

Laboratório de Biociências da Motricidade Humana (LABIMH) marcodalfior@uol.com.br

\section{Max Luciano Dias Ferrão}

Laboratório de Biociências da Motricidade Humana (LABIMH) maxferrao@ig.com.br

\section{Carla Mourão Bittencourt}

Universidade Castelo Branco - UCB - Brasil

Laboratório de Biociências da Motricidade Humana (LABIMH)

carlamestrado@ig.com.br
Daniela Reys Aubaut

Universidade Castelo Branco - UCB - Brasil

Laboratório de Biociências da Motricidade Humana (LABIMH)

daubaut@ig.com.br

Estélio Henrique Martin Dantas

Professor Titular do Programa de Pós Graduação Stricto Sensu em Ciência da Motricidade Humana - UCB - Brasil

Laboratório de Biociências da Motricidade Humana (LABIMH)

Bolsista de produtividade em pesquisa do CNPq.

estelio@cobrase.com.br

DALFIOR, M.A.; FERRÃO, M.L.D.; BITTENCOURT, C.M.; AUBAUT,D.R.; DANTAS, E.H.M. Perfil leucocitário na faixa etária de 15 a 22 anos em função do nível de condicionamento aeróbico. Fitness \& Performance Journal, v.3, n.5, p. 279-283,2004

Resumo: Não se sabe ao certo todas as influências que a atividade física pode ter sobre a imunidade, mas, pesquisas atuais, sugerem que o exercício pode reduzir a incidência e a severidade de infecções, assim como aumentar as funções imunes. A redistribuição dessas células vem sendo atribuída a trocas hormonais ocorridas imediatamente após os exercícios. Sendo assim, o objetivo deste estudo foi verificar as possíveis alterações leucocitárias ocorridas em função do nível de condicionamento cardiopulmonar. A amostra foi constituída de 30 indivíduos, sendo 22 mulheres ( $17 \pm 1,48$ anos) e 8 homens (17 $\pm 1,77$ anos). $\bigcirc$ sangue foi coletado e utilizou-se o hemograma de Shilling (contagem específica de leucócitos); a determinação do $\mathrm{VO}_{2 \mathrm{max}}$. foi obtida através do protocolo de Balke. Pode-se concluir que o condicionamento não influencia diretamente o aumento da capacidade imunológica do indivíduo. Desta forma, os resultados deste trabalho não corroboram outras literaturas que afirmam existir alterações significativas no perfil leucocitário em função do condicionamento físico.

Palavras-chave - Condicionamento aeróbico, Leucócitos, Funções Imunes.

\section{Endereço para correspondência:}

Av Salvador Allende, 6.700 - Recreio dos Bandeirantes - Rio de Janeiro - RJ - CEP 22780-160 
Leukocytic profile in age range 15 - 22 as function of aerobic conditioning

Recent research suggests that exercise might reduce infections occurrence and severity, improving immune functions. Alterations on immune system are connected to hormonal changes, which occurs immediately after exercise. Therefore, the purpose of this study was to verify possible leukocytic alterations due to cadiopulmonary conditioning. Thirty subjects, 22 women (age 17 1, 48 years) and 8 men (17 $\pm 1,77$ years) participated the study. Blood samples were collected, and Shilling hemogram was used to do leukocyte counting. $\mathrm{VO}_{2 \max }$. was determined with Balke protocol. It can be concluded that aerobic conditioning doesn't have direct influence on immune function improvement, for that reason, the results of the present study permit a different position in contrast to other studies, as they verified significant changes on immune cells due to exercise.

Keywords - Aerobic conditioning, Leukocytes, Immune Function.
El perfil leucocitario em la faja de edad de 15 a 22 años en función Del nível de lo condicionamento

El perfil leucocitario en la faja de idad de 15 a 22 años en función del nivel de lo condicionamiento. No se sabe al cierto todas las influencias que la actividad física tiene sobre la inmunidad, todavía, estudios actuales, insinuan que el ejercicio podría amenguar la incidencia e la severidad de las funciones inmunes. La redistribución de las células ha sido atribuida a los cambios hormonales sucedidos inmediatamente después de los ejercicios. Por eso, el objetivo del presente estudio fue verificar las posibles alteraciones leucocitarias sucedidas en función del grado de condicionamiento cardiopulmonar. La muestra fue compuesta de 30 individuos, donde 22 eran mujeres (17 $\pm 1,48$ años) e 8 hombres $(17 \pm 1,77$ años).El sangre fue coletado e utilizó-se el hemograma de Shilling (contagem especifica de los leucocitos). La determinación del $\mathrm{VO}_{2 \text { max }}$, fue adquirida por el protocolo de Balke. Se puede concluir que el condicionamiento no tiene influencia directamente al acrecentamiento de la capacidad inmunológica del individuo. Desta manera, los resultados del trabajo nos dan permiso de sicordar de algunas literaturas que afirman la existencia de alteraciones significativas en el perfil leucocitario en función del nivel de lo condicionamiento.

Palabras clave - Condicionamiento aeróbico, leucocitos, funciones inmunes.

\section{INTRODUÇÃO}

O sistema imune é regulado por uma série de complexas interações, das quais participam principalmente os linfócitos $T$ e $B$, macrófagos e outros componentes, sendo os linfócitos e monócitos as células que participam mais diretamente da resposta imune. Todas as células do sistema imune originam-se de uma célula primordial pluripotente (stem cell), encontrada na medula óssea. A partir desta célula, desenvolvem-se as demais, coletivamente chamadas de leucócitos. (ANTUNES, 1992; ABBAS, 2002; JANEWAY, 2002).

Diversos são os fatores endógenos e exógenos que influenciam o perfil imunológico do ser humano: a variação da temperatura, o estresse, a realização de atividades físicas extenuantes ou até mesmo um banho frio podem provocar modificações. Apesar do exercício físico agir sobre todos os sistemas do organismo, os maiores efeitos incidem sobre o sistema cardiovascular e respiratório, sobre os quais se baseia o condicionamento orgânico (DANTAS, 1992, p. 125). Ao analisar-se o efeito dos programas de condicionamento físico nos parâmetros cardiovasculares, observa-se que o efeito de diferentes tipos e intensidades sobre este sistema é de considerável interesse. $\bigcirc$ condicionamento físico refere-se ao estado de adaptação apresentado pelo organismo, no sentido de responder adequadamente aos esforços físicos de diferentes tipos, intensidades e durações. Kim et al. (2003, p. 102) afirmam que no caso das respostas imunológicas, a intensidade e a duração dos exercícios são fatores importantes para a proporção e manutenção das células imunes.
Até hoje, não se sabe ao certo todas as influências que a atividade física pode ter sobre a imunidade, mas, pesquisas atuais, sugerem que o exercício pode reduzir a incidência e a severidade de infecções, assim como aumentar as funções imunes. A redistribuição dessas células vem sendo atribuída a trocas hormonais ocorridas imediatamente após os exercícios. Partindo deste ponto, pode-se dizer que os exercícios físicos são fundamentais nas trocas hormonais e na alteração e proliferação dos leucócitos, mesmo que isto ocorra apenas imediatamente após a atividade física.

objetivo deste trabalho foi verificar as possíveis alterações leucocitárias ocorridas em função do nível de condicionamento cardiopulmonar.

\section{MÉTODOS}

\section{Sujeitos}

A amostra deste estudo foi constituída de 30 indivíduos aparentemente sadios, sendo 22 mulheres (idade média $17 \pm 1,48$ ) e 8 homens (idade média $17 \pm 1,77$ ).

Precedendo a coleta laboratorial dos dados necessários, o procedimento foi exposto aos sujeitos e, após contar com a autorização dos mesmos, realizou-se o preenchimento de uma ficha de coleta de dados com informações individuais. Em seguida, mediu-se a estatura e aferiu-se a freqüência cardíaca (FC) e a pressão arterial em repouso (PA). 


\begin{tabular}{lccc}
\hline Variável & $\mathrm{F}$ calculado & Significância & Resultado \\
\hline $\mathrm{VO}_{2 \text { max }}$ & 3,942 & $0,0570>0,05$ & iguais \\
$\%$ Linfócitos & 0,532 & $0,4794>0,05$ & iguais \\
$\%$ Monócitos & 0,087 & $0,7733>0,05$ & iguais \\
\hline
\end{tabular}

Tabela 2 - Perfil Leucocitário do Universo Amostral $\left(n^{*}=30\right)$

\begin{tabular}{lccccc}
\hline & Linfócito(\%) & Linf_abs & Monócito(\%) & Mono_abs & Leucócito(total) \\
\hline Média & 34 & 2746,0 & 2 & 193,1 & 8149,0 \\
D. P.** & 10 & 957,44 & 1 & 87,01 & 1204,03 \\
\hline
\end{tabular}

* número total (mulheres e homens)

**desvio padrão

\section{Análise sangüínea}

Inicialmente realizou-se a coleta de sangue dos indivíduos para o estudo laboratorial dos linfócitos e monócitos, visando a determinar a quantidade e qualidade dos glóbulos brancos. Após coleta de sangue por punção venosa do grupo, utilizou-se o hemograma de Shilling (contagem específica de leucócitos). Coletado o sangue, realizados os esfregaços e submetidos à coloração por May-Grunwald Giemsa, segundo as recomendações técnicas usuais, a preparação foi examinada em microscópio com objetiva de imersão (100X) (Hund monocular). Focalizada a preparação, contou-se em zigue-zague 100 elementos em cada lâmina.

\section{Avaliação do condicionamento aeróbico}

O segundo procedimento experimental constituiu-se da determinação do $\mathrm{VO}_{2 \text { max }}$. por meio do protocolo de Balke, descrito por Marins \& Giannichi (1996), onde são empregados estágios múltiplos de 2 minutos com magnitude de 25 Watts, para indivíduos não atletas. Entre os procedimentos para a determinação do $\mathrm{VO}_{2 \max }$. utilizando a fórmula de Balke, verificou-se o peso corporal do avaliado antes da realização do teste, bem como a última carga completada pelo indivíduo em Watts.

\section{Tratamento estatístico}

O nível de significância considerado foi de $p<0,05$, com técnicas de análise descritiva, por média e desvio padrão e, para o teste de inferência, utilizou-se ANOVA one way e o Teste de Correlação de Pearson, a fim de verificar a existência ou não de relação funcional entre as variáveis estudadas.

No sentido de verificar a existência ou não de uma relação funcional entre os leucócitos agranulócitos e a capacidade cardiopulmonar $\left(\mathrm{VO}_{2 \text { max }}\right)$, utilizou-se o teste estatístico de inferência, correlação de Pearson, identificado pelo coeficiente " $r$ de Pearson". Para um grau de liberdade igual a 28 e uma significância de $p<0,05\left(^{*}\right)$, para a correlação ser significativa, o coeficiente $r$ de Pearson deve ser maior ou igual a 0,3494 e, para uma significância $p<0,01 \quad(* *), r$ de Pearson de ser maior ou igual a 0,4486 .

\section{RESULTADOS E DISCUSSÃO}

Neste trabalho será utilizado um único Universo Amostral posto que não foram observadas diferenças significativas entre os sexos, como descrito na Tabela 1. Outros estudos similares também utilizaram ambos os sexos em um mesmo Universo (VENKATRAMAN e PENDERGAST, 1998; PAW et al., 2000).

O Universo amostral apresentou um perfil leucocitário e capacidade aeróbica conforme as Tabelas 2 e 3, respectivamente. $\mathrm{Na}$ Tabela 2, são apresentados os valores relativos e absolutos dos linfócitos e monócitos, e na Tabela 3, foi observado o valor absoluto do $\mathrm{VO}_{2 \max }$. e a capacidade cardiopulmonar; em ambas as tabelas foram apresentadas a média e o desvio padrão.

Correlacionando o índice leucocitário com a capacidade aeróbica, observou-se que não existem correlações significativas $(p<$ $0,05)$ entre os leucócitos agranulócitos absoluto e relativo com a capacidade máxima de absorção de oxigênio $\left(\mathrm{VO}_{2 \max } \cdot\right)$, onde os índices $r$ de Pearson calculados foram $\left(\mathrm{VO}_{2 \max } \times\right.$ \% Linfócitos $\rightarrow r=-0,07)$ e $\left(\mathrm{VO}_{2 \max } \times\right.$ \%Monócitos $\left.\rightarrow r=-0,06\right)$.

Esse resultado concorda com a hipótese de não existir uma melhora no perfil leucocitário, em indivíduos que já apresentam um certo nível de condicionamento aeróbico. Em suma, esse resultado nega uma outra hipótese de existir uma relação funcional de proporcionalidade direta entre o perfil leucocitário, entendida como a contagem relativa e absoluta de linfócitos e monócitos no sangue, com a capacidade máxima de absorção de oxigênio. Por outro lado, a fim de transcenderem-se as possibilidades analíticas das variáveis independentes (linfócitos e monócitos), uma vez que as mesmas foram analisadas isoladamente e não apresentaram correlações significativas com o $\mathrm{VO}_{2 \text { max }}$. fizeram-se, então, combinações algébricas entre as mesmas, obtendo-se como melhor resposta funcional combinada à variável dependente $\mathrm{VO}_{2 \max }$ a razão entre \%Monócitos e \% Linfócitos, conforme os resultados evidenciados na Tabela 4.

Tabela 3 - $\mathrm{VO}_{2}$ Máximo do Universo Amostral $\left(n^{*}=30\right)$

\begin{tabular}{lc}
\hline Geral & $\mathrm{VO}_{2}$ Máximo \\
\hline Média & 25,88 \\
D. P.** & 4,29 \\
\hline
\end{tabular}

* número total (mulheres e homens)

** desvio padrão 


\begin{tabular}{lccc}
\hline Var. Independente & Var. Depend. $\mathrm{VO}_{2 \max }$ & Significância $\mathrm{p}$ & Resultado \\
\hline$\%$ Mono \%Linfoc & $\mathrm{r}=0,36>0,3494$ & $0,0497<0,05$ & Correlacionando \\
\hline
\end{tabular}

Esse resultado indica que a correlação é significativa $(p=0,0497$ $<0,05)$. Todavia, o reduzido tamanho da amostra limita a concluir que existe um forte indicativo de relação funcional entre as variáveis aqui estudadas, uma vez observada a combinação algébrica entre as variáveis \%Monócitos e \% Linfócitos, correspondendo à razão entre as mesmas, combinadas à variável $\mathrm{VO}_{2 \max }$ Como já fora observado anteriormente, essa razão é diretamente proporcional ao $\mathrm{VO}_{2 \max }$. Pode-se concluir que $\circ \mathrm{VO}_{2 \max }$. cresce proporcionalmente ao aumento no número de monócitos e à redução no número de linfócitos, e vice-versa.

As primeiras pesquisas sobre a influência da atividade física sobre o sistema imunológico datam do final do século dezenove com Shultz (1893, apud ZOGAIB, 1986), e no início do século passado com Larrabee (1902, apud LISEN \& UHLENBRUCK, 1992) e Nash (1988), ambos revelando vigorosa leucocitose após uma prova de maratona. As influências da atividade física vêm sendo amplamente estudadas, e sugerem que o exercício pode reduzir a incidência ou a severidade das infecções, assim como aumentar as funções imunes. Inclusive, Pedersen \& Hoffman-Goetz (2003, pp. 1056) afirmam que nos últimos anos, diversos estudos demonstraram que o exercício induz modificações fisiológicas consideráveis no sistema imune.

Natale et al. (2003) realizaram um estudo que submeteu a um programa de treinamento sujeitos moderadamente treinados. Segundo o trabalho realizado, o exercício promove um aumento significativo na contagem total de leucócitos imediatamente após a atividade, seja ela de alta intensidade (máxima) ou moderada (submáxima). Esses resultados foram mais significativos do que os apresentados pelos exercícios de resistência (musculação), embora tanto o exercício aeróbico intenso quanto o de resistência muscular tenham resultado num decréscimo significantemente mais prolongado na relação $\mathrm{CD} 4+/ \mathrm{CD} 8+$ do que o exercício aeróbico submáximo (resultados também encontrados por outros autores, como elucida KIM, 2003). Nesse caso, a leucocitose foi devida principalmente a um aumento na quantidade de neutrófilos e monócitos, embora também tenha ocorrido um pequeno aumento no número de linfócitos. Resultados que indicam que exercícios intensos prejudicam as funções imunológicas, enquanto exercícios moderados elevam a atividade do sistema imune, são encontrados em diversos outros trabalhos (NIEMAN \& NEHLSEN-CANNARELLA, 1991; NEHLSEN-CANNARELLA, 1991; SOLOMON, 1991; LAWLESS et al., 1995; BRINES et al., 1996; SHEPHARD, 1998; SHEPHARD, 2003; NIEMAN, 2000; ROWBOTTON e GREEN, 2000; GOMEZ-MERINO et al., 2003; KAPASI et al., 2003; KIM et al., 2003).

Natale et al. (2003) afirmam que exercícios físicos muito intensos causam dano tecidual, produção de hormônios do estresse e alterações do número e função de diversas células do sistema imune no sangue circulante, o que confirma que "a atividade regular, moderada, está associada a um aumento na resistên- cia, enquanto a inatividade, ou exercícios extremos, está ligada a uma diminuição na resistência imunológica"(BRINES, 1996, p. 252), pois a prática regular de exercícios reduz os riscos cardiovasculares, aumenta a quantidade de vários hormônios circulantes, facilita o controle do peso corporal e reduz o estresse, aumentando a sensação de bem estar. Estas afirmações corroboram com Shephard (2003, p.262), quando este afirma que a atividade física exaustiva afeta a atividade das células NK e dos níveis de imunoglobulinas em indivíduos saudáveis, deixando-os suscetíveis a infecções virais, porém, intensidades moderadas aumentam a atividade imune (NIEMAN, 2000; ROWBOTTON et al., 2000; GOMEZ-MERINO et al., 2003; KAPASI et al., 2003; KIM et al., 2003).

De acordo com Natale et al. (2003), após um período de recuperação de 24 horas, os sujeitos estudados não apresentaram diferenças residuais entre os níveis leucocitários, nas condições de exercício estudadas. Talvez isto explique o fato dos sujeitos deste trabalho não apresentarem uma melhora tão evidenciada no perfil leucocitário, posto que a análise laboratorial não foi realizada imediatamente após o exercício; além disso, os sujeitos eram moderadamente treinados.

\section{CONCLUSÃO}

Com base na análise dos resultados que envolveram a pesquisa dentro dos seus objetivos e limitações, pode-se concluir que o condicionamento não influencia diretamente o aumento da capacidade imunológica do indivíduo. Desta forma, os resultados deste trabalho não corroboram outras literaturas que afirmam existir alterações significativas no perfil leucocitário em função do condicionamento físico. No entanto, os resultados demonstrados apontam que $\circ \mathrm{VO}_{2 \max }$. aumenta proporcionalmente a variação do aumento do número relativo de monócitos e redução do número de linfócitos, e vice-versa. Isso pode ter ocorrido devido ao número da amostra utilizada, e sugere-se a utilização de universos maiores, que transcendam os resultados apontados. Recomenda-se também que sejam utilizados aparatos técnicos laboratoriais de última geração, além de um controle rígido na determinação do condicionamento aeróbico da amostra.

\section{REFERÊNCIAS BIBLIOGRÁFICAS}

ABBAS, Abul K.; LICHTMAN, A. H.; POBER, J. S. Imunologia celular e molecular. $4^{a}$ ed. Rio de Janeiro: Revinter, 2002.

ANTUNES, Lucyr J.; MATTOS, K. T. F. Imunologia médica. São Paulo: Atheneu, 1992.

BRINES, Robert; HOFFMAN-GOETZ, L.; PEDERSEN, B. K. Can you exercise to make your immune system fitter? Immunology Today. v. 17, n.6., 252 - 254. Junho, 1996.

DANTAS, E. H. M. A prática da preparação física. $4^{a}$ ed. Rio de Janeiro: Shape, 1999.

GOMEZ-MERINO, Danielle et al. Immune and hormonal changes following intense military training. Military Medicine. v. 168, n. 12., p. 1034 - 1038. Dezembro, 2003. 
JANEWAY, C. A. et al. Imunobiologia: o sistema imunológico na saúde e na doença. $5^{a}$ ed. Porto Alegre: Artmed, 2002.

KAPASI, Zoher $\mathrm{F}$. et al. Effect of duration of a moderate exercise program on primary and secondary immune responses in mice. Physical Therapy. v.83, n. 7., p. $638-647$. Julho, 2003

$\mathrm{KIM}, \mathrm{H}$. et al. Modulation of immune responses by treadmill exercise in Sprague - Dawley rats. J. Sports Med. Phys. Fitness. v. 43, n 1, p.99 - 104. Março, 2003.

LAWLESS, D. S.; JACKSON, C. G. R.; GREENLEAF, J. E. Exercise and human immunodeficiency virus (HIV - 1) infection. Sports Med. v.19 n.4 p. 235 - 239, 1995.

LISEN, H.; UHLENBRUCK, C. Effects of endurance training on changes in serum glycoproteins and immunoglobulins. Activity Science. v.5, p.189, 1992.

MARINS, J. C. B.; GIANNICHI, R. S. Avaliação e prescrição de atividade física. Rio de Janeiro: Shape, 1996.

NASH, D. A. The leukocytosis of exercise. J. Sports Medicine. v. 10, p. $333-363$ 1988

NATALE, V. M. et al. Effects of three different types of exercise on blood leukocyte count during and following exercise. Sáo Paulo Medical Journal. v. 121, n. 1, p. 9-14, 2003.

NEHLSEN - CANNARELLA, S. L. The effects of moderate exercise training on immune response. Med. Sci. Sports Exerc. V. 10, p. 23 - 45, 1991.

NIEMAN, D. C., NEHLSEN - CANNARELLA, S. L. The effect of acute moderate exercise on leukocyte and lymphocyte subpopulations. Med. Sci. Sports Exerc. v.23, p. 578 $585,1991$.
NIEMAN, D. C. Is infection risk linked to exercise workload? Med. Sci. Sports Exerc. v. 32 , n.7, p.S406 - S411. Julho, 2000

PAW, C. A. et al. Immunity in frail elderly: a randomized controlled trial of exercise and enriched foods? Med. Sci. Sports Exerc. v. 32, n. 12, p. 2005 - 2011, Dezembro 2000.

PEDERSEN, B. K.; HOFFMAN - GOETZ, L. Exercise and the immune system: regulation, integration and adaptations. Physiological Reviews. v.80, n. 3, p. 1055 - 1081. Julho, 2003.

ROWBOTTOM, D. G.; GREEN, K. J. Acute exercise effects on the immune system. Med. Sci. Sports Exerc. V.32, n.7, p. S396 - S405. Julho, 2000.

SHEPHARD, R. J. Exercise, immune function and HIV infection. J. Sports Med. Phys. Fitness. v.38, n.2, p. $101-110$. Junho, 1998.

SHEPHARD, R. J. Adhesion molecules, cathecolamines and leukocyte redistribution during and following exercise. Sports Med. v.33, n.4, p. 261 - 284. 2003

SOLOMON, G. F. Psychosocial factors, exercise, and immunity: athletes, elderly persons and HIV patients. Int. J. Sports Med. v.12, s.1, p. S50 - S52. 1991.

VENKATRAMAN, J. T.; PENDERGAST, D. Effects of the level of dietary fat intake and endurance exercise on plasma cytokines in runners. Med. Sci. Sports Exerc. v.30, n.8, p 1198 - 124. Agosto, 1998.

ZOGAIB, R. Effect of maximal physical exercise on T lymphocyte subpopulations. J. Sports Med. v.8, p. 114 - 117, 1986. 